Esta obra está bajo una Licencia Creative Commons Atribución-NoComercial-Compartirlgual 4.0 Internacional

(c) (1) (8) ()

El panorama español de medios y blogs especializados en I+D+i

Laura Parada Vila y Ana Bellón Rodríguez

DOI: https://doi.org/10.24215/16696581e254

\title{
El panorama español de medios y blogs especializados en $I+d+i$
}

\section{The Spanish media landscape and blogs specialized in $R+D+i$}

\author{
Laura Parada Vila laurigun@gmail.com \\ https://orcid.org/0000-0002-3458-7773
}

España

\section{Ana Bellón Rodríguez ana.bellon@usc.es}

http://orcid.org/0000-0002-8779-1110

Universidad de Santiago de Compostela/Consejo Superior de Investigaciones Científicas, España 


\section{Resumen}

En la última década en España, según datos de la Encuesta de Percepción Social de la Ciencia y la Tecnología, la sociedad incrementó su interés por las informaciones científicas y tecnológicas. En este contexto, en un primer bloque se explora el panorama mediático español para identificar por soportes (prensa, radio, televisión e Internet) algunas de las principales iniciativas a través de las que se está apostando por alfabetizar al público no especializado en Investigación, Desarrollo e Innovación ( $(+D+i)$ e incrementar su cultura científica y tecnológica. En un segundo bloque se explora la blogosfera española especializada en divulgación científica y tecnológica.

Palabras Clave: España; ciencia; tecnología; blogs; divulgación.

Abstract

In the last decade in Spain, according to the Survey of Social Perception of Science and Technology, society increased its interest in scientific and technological information. In this context, in a first part we explore the Spanish media landscape to identify by the media (press, radio, television and Internet) some of the main initiatives through which the media is committed to teaching literacy to the public not specialized in Research, Development and Innovation $(\mathrm{R}+\mathrm{D}+\mathrm{i})$ and increase its scientific and technological culture. In a second part, we explore the Spanish blogosphere specialized in scientific and technological dissemination.

Keywords: España; science; technology; blogs; dissemination.

\section{Introducción}


La Encuesta de Percepción Social sobre la Ciencia y la Tecnología es una iniciativa promovida por la Fundación Española para la Ciencia y la Tecnología (FECYT), entidad pública creada en el año 2001 y dependiente en la actualidad del Ministerio de Ciencia e Innovación. Se realiza con periodicidad bienal desde el año 2002 con el cometido de profundizar en las relaciones entre ciencia, tecnología y sociedad. Desde su creación se han realizado, por tanto, nueve ediciones. Un análisis comparativo de estas ediciones arroja datos sobre cómo ha evolucionado el interés de la sociedad por la I+D+i o cuáles son los principales medios a los que recurre el público general para informarse sobre ciencia y tecnología.

En su última edición (FECYT, 2017) revela que una de cada seis personas $(16,3 \%)$ manifiesta de manera espontánea interés por los temas de ciencia y tecnología. Este dato es similar al de la encuesta realizada en el año 2016 (16\%). En general, continúa siendo mayor entre los hombres $(18,9 \%)$ que entre las mujeres $(13,9 \%)$, observándose una reducción progresiva de esta brecha de género en la última década (2008-2018) con una tendencia al alza entre las mujeres de todas las edades.

Internet es la primera fuente de información científica y tecnológica para un 40,3\% de los ciudadanos (un aumento desde el $37,7 \%$ de la encuesta anterior), pero es la televisión la más consultada cuando se cita más de un medio para estar informado en ciencia y tecnología, con un $75,7 \%$, frente al $63,4 \%$ de Internet. La Red supera a la televisión como fuente de información científica entre personas de 15 a 34 años, siendo también la primera fuente de información para quienes demuestran un interés alto por temas científicos y tecnológicos $(80,3 \%)$, seguida de la televisión $(72 \%)$. Sin embargo, para quienes manifiestan un interés bajo en temas científicos y tecnológicos, la televisión es la primera fuente de información $(78,8 \%)$, frente al $38,2 \%$ de Internet.

El uso de Internet como fuente informativa ha aumentado desde un 52,7\% (FECYT, 2010) a un $63,4 \%$ (FECYT, 2018). Las personas que declaran informarse sobre ciencia y tecnología a través de la Red lo hacen fundamentalmente con las redes sociales $(75,7 \%)$, vídeos $(61,9 \%)$, medios digitales generalistas $(58,9 \%)$, Wikipedia $(52 \%)$, medios digitales especializados en ciencia y tecnología $(44,1 \%)$ y blogs $(35,8 \%)$.

Los blogs, por tanto, ocupan en la actualidad la última posición en el ranking de sitios en la Red para informarse y consumir I+D+i en tono divulgativo. Como explican Xosé López y María Luisa Otero (2007), se trata espacios webs que se inscriben en un proceso de reformación que conduce a la creación de sitios para que el público actúe, experimente y dialogue sobre su propia realidad, sus intereses particulares o sobre sus conocimientos.

José Luis Orihuela (2006) identifica tres etapas en su evolución: «Blogosfera 1.0 (1992-1999), cuando se utilizan para compartir en línea noticias y enlaces de interés, fundamentalmente 
referidos a la evolución de la propia Web; Blogosfera 2.0 (1999-2004), cuando se produce el mayor desarrollo de los blogs, fenómeno masivo que experimenta la incorporación de nuevos elementos como las imágenes y el audio, que enriquecen los posts y Blogosfera 3.0 (2005), con blogs que incorporan el vídeo en su contenido y empiezan a utilizar el tag, etiqueta, para crear categorías temáticas» (pp.78-81).

Al tratar las fuentes de información científica hay que hacer alusión al periodismo especializado. Como expresan Francisco Esteve y Juan Carlos Nieto (2014), es una modalidad de comunicación que facilita el contacto entre los especialistas que trabajan en los diferentes ámbitos, configurándose como un instrumento vertebrador de la sociedad, mientras que como indican Pedro Orive y Concha Fagoaga (1974) se trata de un subsector de la información que se canaliza de modo público y racionalizado a través de los instrumentos de comunicación colectiva. Por su parte, Javier Fernández (1993) destaca que «penetra y analiza la realidad a través de las distintas especialidades del saber, la coloca en un contexto amplio, ofrece una visión global al destinatario y elabora un mensaje periodístico que acomoda el código al nivel propio de cada audiencia, atendiendo sus intereses y necesidades» (p.98) y Rosa Berganza (2005) que «analiza, explica e interpreta procesos con rigurosidad utilizando para ello un lenguaje adaptado a las necesidades del público» (p.78).

Como expresa Luis Ángel Fernández (2000), el responsable de hacer transmisible la información especializada, es decir, el periodista especializado, aparece de forma tímida durante el periodo comprendido entre las dos guerras mundiales, época de gran esplendor para el periodismo informativo ya que primaban los intereses éticos y técnicos frente a intereses políticos y económicos. Esta figura profesional debe tener un dominio de las fuentes instituciones y no institucionales, conocimientos profundos de los saberes de su área, dedicación exclusiva al tema y cualidades especiales relacionadas con el área concreta de su especialidad, así como capacidad de análisis. Hay, por tanto, una serie de conocimientos que se pueden adquirir de forma académica, pero hay otros que solo se alcanzan dentro de la profesión (Consuelo López, 1989, p.7).

Son varias las áreas que se pueden identificar en el periodismo especializado: política, economía, ciencia y tecnología, deportes, sociedad y cultura... El periodismo especializado se enmarca en la denominada Sociedad del Conocimiento; ese conocimiento, ocupa un lugar determinante el relacionado con la Investigación, el Desarrollo y la Innovación, conocida por sus siglas como $\mathrm{I}+\mathrm{D}+\mathrm{i}$.

Parafraseando a Méndez (2007), la transmisión de la información científica y tecnológica se hace de la misma manera que se transmite un contenido económico, político o deportivo: adaptándolo al medio. Se puede considerar que esta transmisión arranca en el momento en el 
que la comunicación de un hecho científico deja de estar reservada exclusivamente a los propios miembros de la comunidad investigadora o a las minorías que dominan el poder, la cultura o la economía y con esa transmisión se favorece que la sociedad sea más democrática y más libre para tomar sus propias decisiones (Manuel Calvo, 1992: p. 26).

Según la opinión de Ana Bellón (2017), para que los medios de comunicación divulguen la I+D+i es fundamental la consonancia de una serie de factores. Ella apunta, por una parte, la especialización de los periodistas que desarrollan su labor en los medios para satisfacer las necesidades de información. Y, por otra parte, el compromiso de la comunidad científica y la labor de los Departamentos de Comunicación de los agentes de los sistemas de I+D+i.

\section{Objetivos y Metodología}

El objetivo principal de esta investigación, de carácter exploratorio-descriptivo, es indagar y presentar a grandes rasgos el panorama mediático español y la blogosfera española desde el punto de vista de su especialización en la divulgación de la l+D+i.

Se busca ofrecer respuesta a las siguientes preguntas:

¿Cómo es, a grandes rasgos, el panorama mediático español especializado en I+D+i? ¿Cuáles son en 2019 las principales iniciativas en cada soporte? ¿Hay algunas apuestas representativas que ya no permanezcan vigentes? ¿Cuáles y en qué medios?

¿Cuáles son en España algunos de los principales blogs creados por y para la divulgación científica? ¿Quiénes son sus promotores? ¿Qué áreas científico-técnicas cubren?

\section{Resultados}

El panorama mediático y la divulgación científica y tecnológica

El soporte impreso y la $\mathrm{I}+\mathrm{D}+\mathrm{i}$

La prensa ocupa el tercer lugar en la lista los principales medios elegidos para acceder a información científica y tecnológica y las revistas de divulgación los últimos puestos (FECYT, 2017).

En el ámbito de los diarios en papel españoles, no se cuenta con secciones específicas, por lo general, dedicadas a la I+D+i y estos temas suelen tener cabida en Sociedad y en áreas temáticas como Mar o Economía. Entre los diarios que han apostado por ofrecer un producto impreso especializado en ciencia y tecnología cabe citar Heraldo de Aragón/Henneo con Tercer Milenio, coordinado por Pilar Perla Mateo. Nació el 8 de junio de 1993 como «una cita semanal 
con la ciencia aplicada, creatividad y empresa». Tras más de 25 años en circulación, continúa distribuyéndose semanalmente en papel, los martes, y la actualización ha pasado a ser diaria en su edición digital y redes sociales. Entre los galardones que ha recibido figura el Premio CSIC de Periodismo Científico (1998) o el Premio Especial del Jurado de los Prismas Casa de las Ciencias (2002).

En cuanto a las revistas impresas mensuales especializadas en I+D+i, cabe citar National Geographic, cuya edición española data de 1997 de la mano del grupo de comunicación RBA; Muy Interesante, fundada en 1981 por Juan Caño y editada por el grupo G+J España y Quo, que se distribuyó en papel desde 1995 hasta 2019 editada por el grupo Hearst España.

\section{El soporte radiofónico y la $\mathrm{I}+\mathrm{D}+\mathrm{i}$}

El medio radiofónico ocupa la cuarta posición en el ranking de las principales fuentes a las que recurre la sociedad para informarse sobre I+D+i (FECYT, 2017).

En la radio privada se localizan espacios y microespacios dentro de programas. Entre ellos, cabe citar:

- $\quad$ Cadena Ser. El viajero cuántico, con una duración aproximada de una hora y dirigido por el periodista Javier Gregori. Se emite dentro del programa La Ventana (de lunes a viernes, de 16.00 a 20.00 horas).

- $\quad$ Cadena Cope. A ciencia y Conciencia y Ciencia. El primero tiene una duración de siete minutos y está dirigido por el científico César Nombela, dentro del programa La linterna (de lunes a viernes, de 19.00 a 23.30 horas). El segundo, conducido por el periodista Jorge Alcalde (Quo), tiene una duración aproximada de 10 minutos y forma parte del magazine La Tarde (de lunes a viernes, de 15.00 a 19.00 horas).

- $\quad$ Onda Cero. Eureka, dentro del programa La rosa de los vientos (sábado de 01.30 a 04.00 horas y domingos de 01.30 a 04.00 horas); La brújula de la ciencia, dentro del magazine La brújula de Juan Ramón Lucas (de lunes a viernes de 20.00 a 23.30 horas) y La ciencia y más, dentro del programa Más de uno, de Carlos Alsina (de lunes a viernes, de 06.00 a 12.30 horas).

En la radio pública conviene hacer alusión a:

- Radio Nacional de España (RNE). El tiempo del tiempo, dedicado a la información meteorológica dentro del magazine No es un día cualquiera (sábados y domingos, de 08.30 a 13.30 horas) y El vecino de la azotea, un microespacio de 10 minutos a cargo del periodista Javier Armentia dentro del programa Esto me suena (lunes a viernes, de 15.00 a 18.00 horas). 
- Radio 5. Secretos del cerebro (jueves a las 11.20 horas); Red abierta (lunes a las 17.47 horas y domingos a las 16.25 horas); Planeta vivo (miércoles a las 15.47 horas) e InquietaMente (miércoles a las 10.47 horas).

- $\quad$ A hombros de gigantes, presentado por Manuel Seara Valero. Comenzó a emitirse el 7 de septiembre de 2007 en Radio 5 y Radio Exterior. En 2012 se trasladó a RNE. En la temporada 2019-2020 se emite los domingos de 16.05 a 17.00 horas.

- $\quad$ Radio 5. Entre probetas (los jueves de 21.30 a 22.00 horas). Se trata de una iniciativa personal de José Antonio López Guerrero, actual presentador y director del programa, que «nace para entretener divulgando la ciencia». Tiene una duración aproximada de 25 minutos.

- $\quad$ Radio 3. Sin distancias (de lunes a domingo, de 05.00 a 06.00 horas), monográfico semanal que los martes se dedica a la I+D+i, y Fallo del sistema (los domingos, de 12.00 a 13.00 horas), donde se aborda el mundo científico y tecnológico relacionándolo con el ocio.

- Radio Exterior. Esta emisora, dirigida a los españoles que están fuera del país, tuvo en antena dos espacios especializados en I+D+i: Vanguardia de la ciencia (1995-2007) y El sueño de Arquímedes (2006-2007), ambos presentados por el científico Ángel Rodríguez Lozano.

En cuanto a la radio online cabe citar Ciencia para escuchar, iniciativa del físico y divulgador científico Ángel Rodríguez Lozano con más de una década de trayectoria. En su web, https://cienciaes.com/, se presenta como «una iniciativa para llegar a todo aquel que sienta inquietud por el estudio de la Naturaleza y las leyes que la gobiernan. Los últimos avances de la investigación científica, los retos de la tecnología o la historia de la ciencia tienen cabida en este espacio, explicados de forma amena y rigurosa».

\section{El soporte televisivo y la I+D+i}

La televisión es el medio que la mayoría de la sociedad española utiliza para estar informada sobre la actualidad científica y tecnológica (FECYT, 2017).

En el ámbito de la televisión pública conviene incidir en que en Televisión Española (TVE) ha sido un referente desde sus inicios en 1956 a través, fundamentalmente, de magazines y los documentales. Entre las iniciativas menos contemporáneas que ya no están en emisión figuran Visado para el futuro (1963-1965), Misterios al descubierto (1966-1970), Redes (1996-2014) o Saber Vivir (1997-2018).

- $\quad$ Redes es un referente de divulgación científica en España. Estaba presentado por los investigadores Eduardo Punset, Wesou Telou y Alberto Jo Lee. El contenido estaba dividido en varias secciones: una entrevista, varios coloquios con los demás colaboradores y un informativo. En 2008 cambió su formato y pasó a denominarse Redes 2.0. Adquirió un estilo 
más sencillo por lo que a partir de entonces el contenido se centró en una entrevista a un reconocido científico y se intercalaban pequeños reportajes. Estuvo en antena durante 18 años, siendo uno de los programas más longevos. Con su última emisión, en mayo de 2014, alcanzó la cifra de 600 programas emitidos en La2.

- $\quad$ Saber vivir. Estuvo en emisión desde 1997 hasta 2009 presentado por Manuel Torreiglesias y concebido como un magazine diario de 75 minutos que se emitía por la mañana. A partir de 2009 dejó de ser un programa independiente y se convirtió en una sección de La mañana de La 1. Esta situación se mantuvo hasta 2017, cuando el programa volvió a emitirse como un espacio propio lunes a viernes de 12.00 a 12.30 horas) presentado por Macarena Berlín. A finales de agosto de 2018 se anunció su cancelación, si bien no estuvo exenta de polémica y llegó a barajarse, ante las críticas, su emisión como una versión reducida.

Entre las iniciativas promovidas desde la televisión pública de ámbito estatal española que continúan en emisión figuran: La Aventura del Saber (1992), Agrosfera (1997), Escarabajo verde (1997), Órbita Laika (2014), Lab24 (2014) o Aquí la tierra (2014).

- La aventura del saber se centra en temas de naturaleza, asuntos sociales, ciencia y tecnología y humanidades. Se compone de entrevistas, reportajes y documentales. Se emite de lunes a jueves en La2 a las 10.00 horas.

- $\quad$ Órbita Laika se caracteriza por tratar de divulgar la ciencia con toques de humor. La primera temporada arrancó el 7 de diciembre de 2014. En febrero de 2020 se ha confirmado que próximamente arranará su sexta temporada, en La2. Estará presentada por Eduardo Sáenz de Cabezón y contará con una nutrida nómina de colaboradores: Marián García, en farmacia y nutrición; Ricardo Moure enbiología; Deborah García Bello en química; Javier Santaolalla en física; Laura Morán en psicología; José Miguel Viñas en meteorología y Xurxo Mariño en neurociencia.

En cuanto a la televisión privada en España, y desde el punto de vista de espacios propios, figuran las siguientes apuestas:

- DMax. Programa ADN Max (domingos de 21.30 a 22.30 horas), del canal de televisión Discovery Max, presentado por Xavier Sardá. Empezó a emitirse el 4 de octubre y terminó el 25 de diciembre de 2015.

- El Hormiguero. Atresmedia. (de lunes a jueves, de 21.45 a 22.30 horas). Programa con una sección de divulgación de la ciencia distinguida con el Premio Tecnología XXI 2018.

Hay, también, una apuesta representativa de televisión online: Indagando.tv. Nace el 16 de junio de 2009 por iniciativa privada (Graziella Almendral) con el objetivo de producir vídeos de 
divulgación, formativos, documentales de gran formato y programas de televisión de divulgación científica y médica.

El soporte online y la I+D+i: ediciones online de diarios y portales

En los últimos años Internet ha avanzado posiciones como canal de acceso a la I+D+i (FECYT, 2017).

En la Red los diarios cuentan con su propia edición online donde, a diferencia de la edición impresa, se han localizado ejemplos de secciones específicas dedicadas a la I+D+i. Se ha realizado una búsqueda en los cuatro periódicos de información generalista más leídos de España según el EGM (El País, El Mundo, La Vanguardia y $A B C$ ) y estos son los resultados:

- $\quad$ Elpais.com (12 secciones, una de ciencia)

- $\quad$ Elmundo.es (11 secciones, una de ciencia y salud y otra de tecnología)

- Lavanguadia.com (12 secciones, ninguna dedicada a la I+D+i)

- $\quad$ ABC.es (13 secciones, una dedicada a ciencia)

En la última década, además, la Red ha sido el soporte elegido para albergar iniciativas creadas por y para la divulgación de I+D+i en formato de portales. Es el caso de:

- $\quad$ Agenciasinc.es. Promovida por la FECYT en 2008. Primera agencia pública de ámbito estatal especializada en información sobre ciencia, tecnología e información en español. Ha recibido el Premio Periodístico Concha García Campoi en la Categoría Prensa Digital (2015) y el Prismas Casa de las Ciencias a la Mejor Web de Divulgación Científica (2014). Todos sus contenidos tienen una licencia Creative Commons 3.0.

- $\quad$ Esmateria.com se puso en marcha en 2012 por los promotores de la sección de ciencia del diario Público. En 2014 se asoció con la web de El País. Se presenta como «la web de noticias de ciencia» y su lema es «Lee, piensa, comparte».

- GCiencia.com. Se creó en 2013 por los periodistas Eduardo Rolland y Pablo López y contó con la beca iProx del Colexio Profesional de Xornalistas de Galicia y Novagalicia Banco. Desde 2015 ha obtenido anualmente el apoyo de la FECYT a través de su Convocatoria de Ayudas para el Fomento de la Cultura Científica y de la Innovación. Se presenta como «O portal da ciencia galega». Obtuvo el Premio Periodismo Digital de Galicia (2015).

En cuanto a los espacios en la blogosfera especializados en $1+D+i$, cabe citar:

- Ciencia de sofá. Puesto en marcha en 2013 por Jordi Pereyra Marí con la intención de «despertar el interés por la ciencia entre el público que está menos familiarizado con ella». Desde el blog se resuelven preguntas y dudas relacionadas con la ciencia. Utiliza un tono directo y personal incluyendo sus propias experiencias, a modo de diario. Entre otros, ha Question, Vol. 1, N. ${ }^{\circ}$ 65, abril 2020. ISSN 1669-6581 
recibido el Premio 20Blogs 2016 como Mejor blog de innovación, ciencia y tecnología y Premio Prismas 2017 en la categoría de mejor sitio web.

- Naukas. Iniciativa que surgió a partir de la colaboración entre Miguel Artime, Antonio Martínez, Javier Peláez y José Cuesta, periodistas y divulgadores científicos que crearon sus propios blogs sobre ciencia y que en 2010 vieron en la unión de un proyecto el futuro para avanzar en la divulgación. Así, promovieron en conjunto el blog Amazing.es, que se convirtió en una de las plataformas referentes en divulgación científica en España. Tal fue la repercusión que consiguió que el dominio inicial evolucionó hasta el actual Naukas. Cuenta con la colaboración de más de 100 profesionales de la ciencia. Además del contenido que se puede encontrar en la web, Naukas también organiza eventos por toda la geografía española con el objetivo de divulgar la I+D+i.

- Ciencia para llevar. Iniciativa del Consejo Superior de Investigaciones Científicas (CSIC) puesta en marcha por el Área de Cultura Científica en el año 2014. Se presenta como «un blog colectivo en donde hablan de los avances y las curiosidades de la ciencia». Se encuentra albergado en el diario digital 20minutos.es. Bajo el nombre colectivo de Mar Gulis (en homenaje a la gran investigadora y divulgadora Lynn Margulis), escriben varias personas dedicadas a la divulgación en el CSIC.

- Chica Geek. Nació en 2007 por iniciativa de Elena Santos para «reivindicar que a las chicas también les interesa la tecnología y luchar contra la imagen frecuentemente negativa que dan algunos medios de comunicación sobre tecnología». En 2016 consiguió el Premio Bitácoras al mejor blog de tecnología.

- Dimetilsulfuro. Blog de la química y divulgadora científica Deborah García Bello. El espacio está especializado en el binomio ciencia-arte, tratando de explicar estos conceptos a través de obras de arte conocidas para tratar de descubrir cuál es la conexión entreambas disciplinas. Obtuvo el Premio Bitácoras (2014) como mejor blog de ciencia y el Premio Prismas (2017).

- Radical Barbatilo. Blog de divulgación científica del bioquímico Jesús Gil. Su cometido fundamental pasa por dar a conocer distintos eventos en los que el autor participa.

- $\quad$ Fogonazos. Blog del periodista y divulgador científico Antonio Martínez Ron. Puesto en marcha en 2003, en 2013 entró a formar parte de la familia de blogs de la revista científica Quo. Su objetivo pasa por fomentar la concienciación de la importancia de la ciencia en la sociedad con un lenguaje claro y directo

- Blog de la Asociación Española de Comunicación Científica (AECC), entidad fundada en 1975 por Manuel Calvo Hernando cuyo cometido es la divulgación de la ciencia a través de la promoción de informaciones científicas en los medios de comunicación, facilitar el diálogo y 
las relaciones con las fuentes de información y velar por la independencia y la objetividad de los contenidos informativos y de libertad de expresión. Desde 2007 la entidad cuenta con un blog, accesible desde su página web, donde sus asociados explican y dan su opinión sobre temas científicos y tecnológicos.

- La cuadratura del círculo. Iniciativa del CSIC promovida desde Andalucía albergada en Eldiario.es.

\section{Conclusiones}

Fruto de este trabajo de investigación exploratorio-descriptivo se ha constatado que en España se está apostando por la divulgación científica y tecnológica a través de los medios de comunicación en mayor o menor medida y con diferencias según los soportes; que el acercar al público general la $\mathrm{I}+\mathrm{D}+\mathrm{i}$ en tono divulgativo puede constituir, incluso, una oportunidad de emprendimiento, y que la vocación de personal dedicado a la ciencia y la tecnología les lleva también a abrir espacios en la blogosfera para contar, en un tono ameno y próximo, la magia que se esconde en los laboratorios.

Como indican Falk y Dierking (2010), dado que la mayor parte de la educación científica de una persona se hace fuera de un ambiente formal, la mejor manera de aumentar la comprensión del público en relación a la ciencia está en el restante de su vida. Es ahí, en el tiempo de libre de las personas, en el tiempo de ocio, donde los medios de comunicación están llamados a jugar un papel clave. Primero, en la alfabetización científica y tecnológica de la sociedad del siglo XXI, es decir, en completar y complementar la formación que se recibe en el ámbito educativo a través de las diferentes etapas de la vida. Después, en el incremento de la cultura científica y tecnológica de una sociedad que ya contaría con una base de conocimiento sobre I+D+i que le permitiría comprender y tener interés en los principales avances y retos a los que se enfrenta la ciencia y la tecnología.

Los medios de comunicación, ese cuarto poder del que tanto se tiene hablado, ni pueden ni deben permanecer ajenos a la necesidad y demanda social de $\mathrm{I}+\mathrm{D}+\mathrm{i}$ en tono divulgativo $\mathrm{y}$ comprensible por el público general, contando para ello con la inestimable colaboración, como se ha puesto de manifiesto por el perfil de promotores de espacios, sobre todo en el ámbito audiovisual y en la blogosfera, de la propia comunidad científica y tecnológica.

\section{Referencias bibliográficas}


Bellón Rodríguez, A. (2017). Las siglas I+D+i en la televisión tradicional y online en España. El caso de Indagando TV. Revista de la Asociación Española de Investigación de la Comunicación, 4 (7), 119-129.

Berganza, M.R (2005). Periodismo Especializado. Pamplona, España: Ediciones Internacionales Universitarias.

Calvo, M. (1992). Periodismo científico. Madrid, España: Editorial Paraninfo.

Esteve Ramírez, F. y Nieto Hernández, J.C. (2014). Nuevos retos del periodismo especializado. Madrid, España: Schedas.

Falk J. H. y Dierking, I. D. (2010). The 95 per cent solution. American Scientist. Recuperado de http://www.americanscientist.org/issues/feature/2010/6/the-95-percent-solution

Fernández del Moral, J. (1993). Fundamentos de la información periodística especializada. Madrid, España: Síntesis.

Fernández Hermana, L. A (2000). Información: universo en expansión o agujero negro. En Comunicar la ciencia en el siglo XXI: I Congreso sobre Comunicación Social de la Ciencia (pp. 185-192). Granada, España: Parque de las Ciencias.

Fundación Española para la Ciencia y la Tecnología (2017). IX Encuesta de Percepción Social de la Ciencia y la Tecnología. Madrid, España: FECYT. Recuperado de https://www.fecyt.es/es/noticia/principales-resultados-de-la-encuesta-de-percepcionsocial-de-la-ciencia-2018

López García, X. y Otero López, M. (2007). Bitácoras: la consolidación del ciudadano. A Coruña, España: Netbiblo.

López Vila, C (1989). La especialización en el periodismo. Periodistas, 27, 6-13.

Méndez, E. (2007). La ciencia como noticia: estrategias discursivas y textuales. La clonación terapéutica. En Delgado Cobos, I. \& Puigvert Pcal, A. (Eds), Exadmiratione et atmicia: homenaje a Ramón Santiago. Madrid, España: Ediciones el Orto.

Orive, P. y Fagoaga, C. (1974). La especialización en el periodismo. Madrid, España: Dossat.

Orihuela, J.L. (2006). La revolución de los blogs. Cuando las bitácoras se convirtieron en el medio de comunicación de la gente. Madrid, España: La Esfera de los Libros. 